\title{
Correction to: Development of efficient and robust Eikonal solver variants for first-arrival seismic modeling
}

\author{
Zagid Abatchev $^{1,2}$ (I) $\cdot$ Gary Binder ${ }^{2} \cdot$ Paul Davis $^{1}$
}

Published online: 16 April 2021

(C) Springer Nature Switzerland AG 2021

\section{Correction to: Computational Geosciences https://doi.org/10.1007/s10596-020-10010-5}

The original version of the article unfortunately contained the following errors:

Incorrectly formatted references that were included in the abstract are now removed. Correct citations are now found in the body of the text available online.

A link to the source code for FMM-VFD was omitted. A section was added at the end titled "Code Availability" with a single sentence "Code used in this study is freely available at https://github.com/abatchev/FMM-VFD "

In Table 1, abbreviations in parentheses were moved to the same line as the model (ie "Shortest Path Method (SPM) ". Without visible cells, this makes it more obvious that each model has 2 line descriptions.
A typo was corrected on lines 10/11; "non non-unique solutions" has been corrected to "non-unique solutions". A typo was corrected on line 124; "lowers" has been corrected to "lower".

A typo was corrected on line 444; "neigborhood" has been corrected to "neighborhood".

A typo was corrected on line 461; "heterogenous" has been corrected to "heterogeneous".

A typo was corrected on line 485; "T his" has been corrected to "This".

Several instances of "numerical" were changed to "numeric" for consistency.

Publisher's note Springer Nature remains neutral with regard to jurisdictional claims in published maps and institutional affiliations.
The online version of the original article can be found at https://doi.org/ $10.1007 /$ s $10596-020-10010-5$

Zagid Abatchev

abatchev@ucla.edu

1 University of California, Los Angeles, 1703 Geology, Los Angeles, CA 90095, USA

2 Automated Seismics LLC, 18345 Sierra Hwy, Suite 7, Santa Clarita, CA 91351, USA 